\title{
Heat Transfer in MHD Ekman Layer on a Porous Plate (").
}

\author{
S. NARASTMHA MURTY
}

Department of Mathematics, College of Science, University of Mosul - Mosul, Iraq

R. K. Prabhakar Rai

Department of Physics, National College, Bangalore University - Bangalore-4, India

(ricevuto il 31 Gennaio 1978)

\begin{abstract}
Summary, - A steady asymptotic solution for the temperature distribution in the case of flow past a porous plate in a rotating frame of reference is obtained. In particular, the temperature distribution for MHD Ekman layer on a porous flat plate is studied. It is seen that, while a steady asymptotic solution is possible in case of suction, no steady temperature field is possible in case of blowing. Further, from the results it is observed that suction and magnetic field have opposing influence on the rate of heat transfer.
\end{abstract}

\section{1. - Introduction.}

When a vast expanse of viscous liquid bounded by an infinite flat plate is rotating about an axis normal to the plate, a layer is formed near the plate where the viscous and Coriolis forces are of the same order of magnitude. This si known as Ekman layer ( $\left.{ }^{1}\right)$. The effect of porosity and of the applied magnetic field on such a layer has been recently investigated by present authors $\left({ }^{2}\right)$ and

(*) To speed up publication, the anthors of this paper have agreed to not receive the proofs for correction.

(1) K. Prandti: Essentials of Fluid Dynamics, 3rd edition (London, 1949), p, 356.

$\left({ }^{2}\right)$ S. Narasimha Murthy and R. K. Prabhakar Ram: Nuovo Cimento, 28 B, 296 (1975). 
the chief motivation of the problem was also discussed in detail by them. The purpose of the present investigation is to extend the work of the present authors to the heat transfer characteristics of the Ekman layer over a flat plate which is maintained at a temperature higher than that of the surrounding stream.

The present motivation comes from a desire of understanding, at least qualitatively, the effect of porosity on the temperature distribution and its role on the boundary layer likely to exist at the core-mantle interface of the Earth where rotation and magnetic-field effects are simultaneously present.

\section{2. - Formulation of the problem.}

For the sake of continuity, we recast in brief the problem discussed by the present authors ( $\left.{ }^{2}\right)$.

A Cartesian co-ordinate system rotates uniformly with angular velocity $\Omega$ about the $z$-axis, taken positive in the vertically upward direction with the plate coinciding with the plane $z=0$ and being maintained at a temperature presently higher than that of the surrounding stream and being uniformly porous. The liquid is permeated by a uniform vertical magnetic field $B_{0}$. The basic equations of the motion are

$$
\begin{aligned}
-2 v \Omega-w_{0} \frac{\mathrm{d} u}{\mathrm{~d} z} & =-\frac{\sigma B_{0}^{2}}{\varrho}(u-v)+\frac{\mathrm{d}^{2} u}{\mathrm{~d} z^{2}}, \\
2(u-v) \Omega-w_{0} \frac{\mathrm{d} v}{\mathrm{~d} z} & =-\frac{\sigma B_{0}^{2}}{\varrho} v+\frac{\mathrm{d}^{2} v}{\mathrm{~d} z^{2}} .
\end{aligned}
$$

The above equations are to be solved subjected to the following boundary conditions :

$$
u=v=0 \quad \text { at } z=0, \quad u \rightarrow U, \quad v \rightarrow 0 \quad \text { as } z \rightarrow \infty .
$$

The solution of the above equations subjected to above conditions (1.3) is given by $\left({ }^{2}\right)$ as

$$
\begin{aligned}
& \frac{u}{U}=1-\exp [-\alpha \eta] \cos \beta \eta, \\
& \frac{v}{U}=\exp [-\alpha \eta] \sin \beta \eta,
\end{aligned}
$$

where

$$
\left\{\begin{array}{l}
\alpha=S+\left[\frac{\left\{\left(4 N+S^{2}\right)^{2}+16 E^{2}\right\}^{2}+\left(4 N+S^{2}\right)}{8}\right], \\
\beta=\left[\frac{\left\{\left(4 N+S^{2}\right)^{2}+16 E^{2}\right\}^{1}-\left(4 N+S^{2}\right)}{8}\right] .
\end{array}\right.
$$


Here

$$
\begin{aligned}
& S=\frac{w_{0}}{U_{\infty}} \text { is the suction parameter, } \\
& N=\frac{\sigma B_{0}^{2} \nu}{\varrho U_{\infty}^{2}} \text { is the magnetic parameter, } \\
& E=\frac{2 \Omega \nu}{U_{\infty}^{2}} \text { is the rotation parameter. }
\end{aligned}
$$

Now the equation that governs the heat transfer is the energy equation

$$
-\varrho c_{p} w_{0} \frac{\mathrm{d} T}{\mathrm{~d} z}=\lambda \frac{\mathrm{d}^{2} T}{\mathrm{~d} z^{2}}+\varrho v\left[\left(\frac{\mathrm{d} u}{\mathrm{~d} z}\right)^{2}+\left(\frac{\mathrm{d} v}{\mathrm{~d} z}\right)^{2}\right],
$$

where $\varrho$ is the density, $c_{p}$ is the specific heat at constant pressure, $w_{0}$ is the suction or injection velocity, $\lambda$ is the thermal conductivity.

Introducing the following diemensionless quantities:

$$
\begin{aligned}
& \theta(\xi)=\frac{T-T_{0}}{T_{\infty}-T_{0}}, \quad E_{\mathrm{c}}=\frac{U_{\infty}^{2}}{c_{p}\left(T_{0}-T_{\infty}\right)} \quad \text { Eckert number } \\
& P_{\mathbf{r}}=\frac{\rho c_{\mathfrak{p}} \nu}{\lambda}, \quad S=\frac{w_{0}}{U_{\infty}}
\end{aligned}
$$

and eqs. (1.4) and (1.5) in eq. (1.7), we get

$$
\frac{\mathrm{d}^{2} \theta}{\mathrm{d} \xi^{2}}+P_{\mathrm{r}} S \frac{\mathrm{d} \theta}{\mathrm{d} \xi}=E_{\mathrm{c}} P_{\mathrm{r}}\left(\alpha^{2}+\beta^{2}\right) \exp [-2 \alpha \xi]
$$

where $T_{0}$ and $T_{\infty}$ are temperatures of the plate and of the free stream, respectively, as we assume $T_{0}>T_{\infty}$, and, therefore, the Eckert number is positive.

The boundary conditions for $\theta(\xi)$ are

$$
\theta(0)=0, \quad \theta(\infty)=1
$$

The solution of eq. (1.8) satisfying (1.9) is

(1.10) $\theta(\xi)=1-\exp \left[-S P_{\mathrm{r}} \xi\right]+\frac{E_{\mathrm{c}} P_{\mathrm{r}}\left(\alpha^{2}+\beta^{2}\right)}{2 \alpha\left(S P_{\mathrm{r}}-2 \alpha\right)}\left(\exp \left[-\delta P_{\mathrm{r}} \xi\right]-\exp [-2 \alpha \xi]\right)$ for $S P_{\mathrm{r}}-2 \alpha \neq 0$ and

$$
\theta(\xi)=1-\frac{1+E_{\mathrm{c}} P_{\mathrm{r}}\left(\alpha^{2}+\beta^{2}\right)}{S} \exp [-2 \alpha \xi]
$$

whenever $S P_{\mathrm{r}}-2 \alpha=0$. 
Now the rate of heat transfer at the plate is given by

$$
q_{w}=-\lambda\left(\frac{\mathrm{d} T}{\mathrm{~d} z}\right)_{z=0}=\frac{\lambda U_{\infty}}{\left(T_{0}-T_{\infty}\right) \nu}\left(\frac{\mathrm{d} \theta}{\mathrm{d} \xi}\right)_{\xi=0} .
$$

Using eqs. (1.10) and (1.11) in (1.12) yields

$$
\left(\frac{\mathrm{d} 0}{\mathrm{~d} \xi}\right)_{\xi-0}=P_{\mathrm{r}}\left[S-\frac{E_{\mathrm{o}}\left(\alpha^{2}+\beta^{2}\right)}{2 \alpha}\right] .
$$

Since $T_{0}>T_{\infty}$, it follows from (1.12) and (1.13) that heat will flow from the plate to the liquid if

$$
S>E_{\mathrm{o}} \frac{\alpha^{2}+\beta^{2}}{2 \alpha},
$$

while heat will flow from the liquid to the plate if

$$
S<E_{0} \frac{\alpha^{2}+\beta^{2}}{2 \alpha} .
$$

Further there will be no heat transfer to or from the wall when

$$
S=E_{\mathrm{c}} \frac{\alpha^{2}+\beta^{2}}{2 \alpha} .
$$

The inequality (1.15) leads to

$$
\begin{aligned}
\left(1-\frac{E_{\mathrm{c}}}{4}\right) S^{2}+\frac{2-E_{\mathrm{o}}}{2 \sqrt{2}} S\left[\left\{\left(4 N+S^{2}\right)^{2}+16 E^{2}\right\}^{\frac{1}{2}}+\left(4 N+S^{2}\right)\right]^{1} & < \\
& <\frac{E_{\mathrm{c}}}{4}\left[\left(4 N+S^{2}\right)^{2}+16 E^{2}\right]^{t}
\end{aligned}
$$

which can be written as

$$
E_{\mathrm{o}}>A / B
$$

where

$$
\begin{aligned}
& A=S^{2}+S \frac{\left[\left\{\left(4 N+S^{2}\right)^{2}+16 E^{2}\right\}^{\ddagger}+\left(4 N+S^{2}\right)\right]^{\ddagger}}{\sqrt{2}}, \\
& B=\frac{S^{2}}{4}+\frac{\left\{\left(4 N+S^{2}\right)^{2}+16 E^{2}\right\}^{\}}}{4}+\frac{S}{2 \sqrt{2}}\left[\left\{\left(4 N+S^{2}\right)+16 E^{2}\right\}+\left(4 N+S^{2}\right)\right]^{\ddagger}
\end{aligned}
$$

Further

$$
P_{\xi}^{-1}\left(\frac{\mathrm{d} \theta}{\mathrm{d} \xi}\right)_{\xi-0}=\left[S-\frac{E_{0}\left(\alpha^{2}+\beta^{2}\right)}{2 \alpha}\right] .
$$




\section{3. - Discussion of the results.}

It is interesting to note from eq. (1.15) that heat may flow from the liquid to the plate even if the plate temperature is higher than the free-stream temperature. This is possible when increased viscous dissipation near the plate may lead to a temperature distribution in its neighbourhood greater than $T_{0}$ despite the fact $T_{0}>T_{\infty}$. If $E_{\mathrm{c}}>4$, inequality (1.17) shows that heat always flows from the liquid to the plate.

Figures 1 and 2 show the values of the dimensionless parameter as given by eq. (1.18) at the plate for values of $S=1,0.5, N=0,5, E^{2}=4,1$ against $E_{\mathrm{o}}$.

From fig. 1 and 2 it is observed that the effect of the increase in the suction parameter for given $N$ and $E^{2}$ is to cause a considerable increase in the rate of heat transfer for a given $E_{\mathrm{c}}$, thereby increasing the value of $E_{\mathrm{c}}$ at which the heat transfer rate becomes zero.

For given $S$ and $E^{2}$, we see that the effect of the magnetic field alone is to decrease the rate of heat transfer for a given $E_{\mathrm{c}}$. Further it decreases the value of $E_{c}$ at which the heat transfer rate changes sign.

In the case of fixed $S$ and $N$ we see that an increase in the rotation parameter causes a decrease in the rate of heat transfer for a given $E_{\text {c }}$ and a decrease in the value of $E_{\mathrm{c}}$ at which the rate of heat transfer changes sign.

Thus we see that the suction and magnetic effects have opposing influence

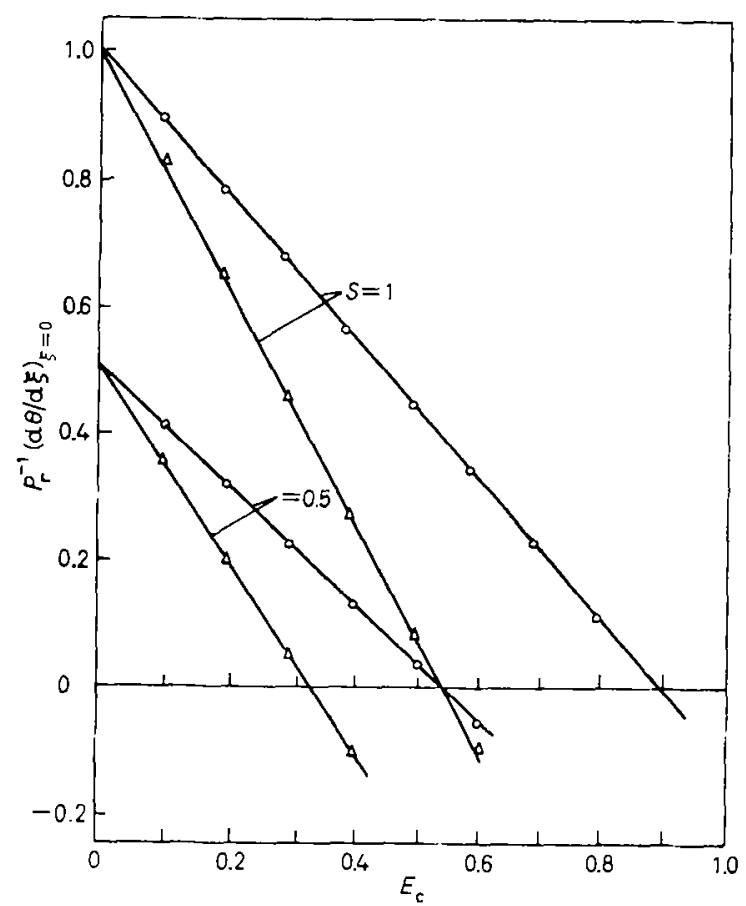

Fig. 1. $-\circ N=0, E^{2}=1 ; \Delta N=5, E^{2}=1$. 


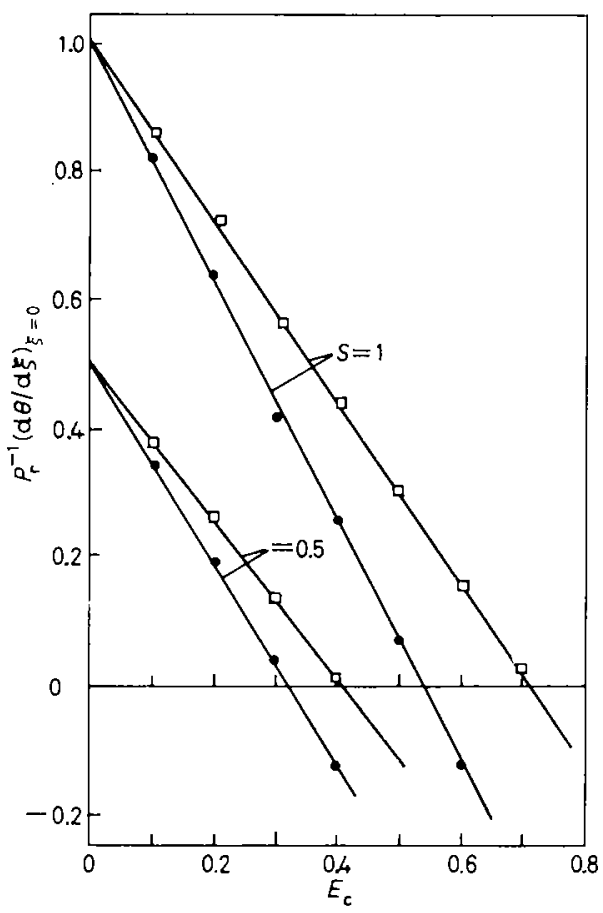

Fig. 2. $-\square N=0, H^{2}=4 ; \bullet N=5, E^{2}=4$.

on the rate of heat transfer. Furthermore, the magnetic and rotation effects alone are a decrease of the rate of heat transfer from the plate. It is interesting to note that the steady asymptotic solution for the tempeature is possible only for the case of suction at the heated wall. But, for the case of blowing, no steady-temperature field is possible since the liquid at infinity is progressively heated both by diffusive processes and by convection of heat towards infinity by blowing.

\section{- RIASSUNTO(*)}

Si ottiene una soluzione asintotica stazionaria per la distribuzione della temperatura nel caso di flusso attraverso una piastra porosa in un sistema di riferimento ruotante. Si studia, in particolare, la distribuzione della temperatura per lo strato MHD di Ekman su una piastra porosa piatta. Si vede che, mentre una soluzione asimptotica stazionaria d̀ possibile in caso di suzione, nessun campo stazionario di temperatura è possibile in caso di insufflazione. Inoltre, dai risultati si osserva che la suzione e il campo magnetico hanno influenza opposta sulla velocità del trasferimento di calore.

(*) Traduzione a cura della Redazione. 